\title{
Dynamic Monte Carlo Simulation of Gelation with Extensive Cyclization ${ }^{\dagger}$
}

\author{
Stephen E. Rankin ${ }^{\ddagger}$, Leo J. Kasehagen ${ }^{\S}$, \\ Alon V. McCormick, and Christopher W. Macosko* \\ Department of Chemical Engineering and Materials Science \\ University of Minnesota, Minneapolis, MN 55455 \\ RECEIVED \\ AUG 172000 \\ OSTI \\ Abstract
}

\begin{abstract}
Recent investigations have implicated cage-like precursors in the unusually high gelation conversion $(\sim 82 \%)$ of acid-catalyzed tetraethoxysilane. However, the statistical models used so far cannot capture kinetic or composition-dependent features of alkoxysilane polycondensation. Here we take a first step towards unified modeling of the kinetics and structure of silica gelation. Dynamic Monte Carlo simulations [J. Šomvársky and K. Dušek, Polym. Bull. 1994 33:369] are developed which permit competition between extensive cyclization and growth. The model includes well-established kinetic trends (hydrolysis pre-equilibrium and first shell substitution effects). As a first approximation, unimolecular-like terms for cyclization reactivity follow the experimental pattern of bimolecular rate coefficients. The present simulations allow unlimited formation of 3-site rings, giving rise to many structures which are not those of real silicates (where 4-site rings dominate). However, the level of cyclization (both cycles per molecule and per site) is consistent with that of real silicates, and is enough to delay gelation to $82 \%$ conversion or higher. These simulations also display a broader range of gelation behavior than prior kinetic models. At high to moderate monomer concentrations, competition between cyclization and growth causes the expected delay of gelation. Upon further dilution, we discover a third regime, absent from prior kinetic gelation models but important for siloxanes: formation of a distribution of polycyclic precursors which still retain enough functionality to gel.
\end{abstract}

†Dedicated to Dr. Karel Dušek in honor of his contributions to polymer science and his $70^{\text {th }}$ birthday, and to the memory of Douglas R. Miller.

${ }^{\ddagger}$ Current address: Sandia National Labs, 1001 University Blvd. SE, Ste. 100, Albuquerque, NM 87106

${ }^{\S}$ Current address: Elf Atochem N.A., Inc., 900 First Ave., King of Prussia, PA 19406

*To whom correspondence should be addressed. 


\section{DISCLAIMER}

This report was prepared as an account of work sponsored by an agency of the United States Government. Neither the United States Government nor any agency thereof, nor any of their employees, make any warranty, express or implied, or assumes any legal liability or responsibility for the accuracy, completeness, or usefulness of any information, apparatus, product, or process disclosed, or represents that its use would not infringe privately owned rights. Reference herein to any specific commercial product, process, or service by trade name, trademark, manufacturer, or otherwise does not necessarily constiture or imply its endorsement, recommendation, or favoring by the United States Government or any agency thereof. The views and opinions of authors expressed herein do nct necessarily state or reflect those of the United States Government or any agency thereof. 


\section{DISCLAIMER}

Portions of this document may be illegible in electronic image products. Images are produced from the best available original document. 


\section{Introduction}

Cyclization plays an unusually important role in siloxanes. Not only can stable single rings be isolated from di(organo)dichlorosilane hydrolytic polycondensation products, ${ }^{1}$ but polycyclic silsesquioxanes $\left(\mathrm{RSiO}_{3 / 2}\right)$ can be recrystallized from the products of trifunctional silane polycondensation, ${ }^{2}$ and polycyclic species can be isolated from soluble silicate solutions by passivation (trimethylsilylation). ${ }^{3}$ More recently, metastable cyclic and polycyclic intermediates have been observed by ${ }^{29} \mathrm{Si}$ nuclear magnetic resonance during synthesis of siloxanes from multifunctional alkoxysilanes. ${ }^{4-6}$ Cycles are kinetically promoted by the bond and torsion angles of siloxane bonds, which favor bent conformations with chain ends relatively close together. ${ }^{7,8}$ Quantum mechanical calculations also suggest that these cycles and polycycles may be thermodynamically favored over linear and branched species. ${ }^{9,10}$

Polycyclic siloxanes are of growing interest as precursors to novel organic-inorganic hybrids, ${ }^{11}$ but they also are frequently a nuisance delaying or preventing gelation of branched siloxanes,${ }^{12}$ silicic acid, ${ }^{13}$ and certain $\alpha, \omega$-Bis(triethoxysilanes).${ }^{14}$ Even in ethoxysilane systems able to gel, four-site rings have been shown by x-ray diffraction to be a prominent structural unit. ${ }^{15,16}$ Adjustable parameters (including monomer concentration, $\mathrm{pH}$, and water content ${ }^{17}$ ) influence the degree and type of cyclization, but so far a quantitative connection between these parameters and the formation and structure of the gel has not been made. This connection will be valuable in understanding and directing the synthesis of silicates.

There has been progress in modeling the formation of siloxanes with cycles, but no single model has so far been consistent with all observed features of silica gelation. A two-stage statistical model was developed by $\mathrm{Ng}$ et al. ${ }^{18}$ (random branching of precursors formed quickly and selectively during the initial stages of polycondensation) that predicts siloxane bond conversions approaching the unusually high value measured for tetraethoxysilane ( $82 \%) .{ }^{18}$ This conversion contrasts strongly the classical Flory ${ }^{19}$-Stockmayer ${ }^{20,21}$ prediction of $33 \%$ for a tetrafunctional monomer. Single-ring precursors predict an $\alpha_{g e l}$ (gel conversion) value of 
up to $60 \%$, but if the precursor is a compact cage-like species such as a prismatic hexamer, $\alpha_{g e l}$ can be as high as $80 \% .^{18}$

While this statistical model illustrates that polycyclic species are essential for a model to match the high gel point of tetraethoxysilane, it is not complete. It does not account for the polydispersity of species observed prior to gelation, ${ }^{22,23}$ and does not contain any adjustable parameters allowing us to model the sensitivity of gel time and structure to synthesis conditions. Both of these problems are related to the absence of kinetics in the model (or at least not accounting for the competition between cyclization and growth).

The second direction taken towards accurately modeling structure development from alkoxysilanes is kinetic. Cyclization rate coefficients for the formation of small, single-ring siloxanes (containing three or four silicon sites) have been measured in systems starting from ethoxysilane monomers. ${ }^{17,23}$ These parameters are found by fitting to ${ }^{29} \mathrm{Si}$ NMR data, but precise data about cycle membership are available only up to limited conversions - well below the gel point.

We first attempted to bridge these two approaches by slightly extending the latter kinetic approach. The revised model preserves reactions forming small (3- and 4- membered) siloxane rings early in reaction and allows those rings to enter into large oligomers (and, eventually, a gel) without further cyclization. ${ }^{24}$ This model remains consistent with the kinetics measured early in reaction, but unfortunately, using either kinetic-recursive or Monte Carlo modeling, the predicted gel points (53\%) still fall short of the experimental value (82\%). ${ }^{24}$ The additional inability to fit data over a large conversion range tells us that this model does not allow enough cyclization to occur. ${ }^{25}$

Here, we extend our kinetic approach even further to more accurately predict structure development and gelation behavior. In the next section, we will describe a model in which unlimited cyclization to form three-membered rings is allowed. These rings are less prevalent than four-membered rings in real silicates, but we use them in this first investigation to learn how extensive cyclization affects gelation. As we will discuss below, four-membered 
rings are conceptually similar to three-membered rings but significantly more difficult to implement. Our model includes specific unimolecular-like reaction terms for the reaction between any two silicon sites with a two-bond path between them. The governing equations and molecular weight distribution are simultaneously solved by the dynamic Monte Carlo simulation technique introduced to polymer science by Dušek and coworkers. ${ }^{26,27}$ These simulations are uniquely capable of showing the structural implications of kinetic models without additional assumptions. ${ }^{28-30}$ While our model incorporates features of alkoxysilane polymerization discovered over the last decade, this is the first kinetic model able to predict a gel conversion as high as the experimentally observed value for tetraethoxysilane.

\section{Dynamic Monte Carlo Approach}

The random branching theory developed by Flory ${ }^{19}$ and Stockmayer ${ }^{20,21}$ provides an extremely powerful framework for understanding gelation. In the theory, reactivities of all functional groups are assumed to be equal throughout polymerization and cyclization is neglected. For ethoxysilanes, however, NMR investigations show that condensation reactivity drops as siloxane bonds are added to monomers ${ }^{17,31,32}$ (in other words, there is a first-shell substitution effect) and that small rings form in abundance. .,17,18,25 $^{2}$

Perturbations exist which allow statistical models to be constructed taking into account first-shell substitution effects ${ }^{33,34}$ and cyclization. ${ }^{35}$ In the perturbation for cyclization, sites which are members of a ring are treated as independent. This means that only the consumption of functional groups by cyclization is captured, and not correlations between sites in rings. These correlations can be captured by instead defining new (cyclic) species which form and become part of larger polymers. ${ }^{18,24}$ This approach to cyclization is not well suited for the polycyclic species present in silicates, ${ }^{18}$ however, for reasons illustrated by Figure 1. To construct a statistical model for silica gelation with polycyclic species, we require the concentrations of those polycycles. Figure 1 shows all possible routes for the formation of the smallest known ${ }^{36}$ polycyclic silicate, the bicyclic pentamer. Differential equations must 
be written for the concentrations of all 13 species in the Figure if we are to accurately model the kinetics of forming the bicyclic pentamer. It is not possible to accurately decompose the bicyclic pentamer into smaller fragments. The same thing is true of larger polycyclic oligomers, for which the number of pathways and isomers increases rapidly. $\mathrm{Ng}$ et al. ${ }^{18} \mathrm{ex}-$ amined the simplified case where all monomers, regardless of the path taken, form a single polycyclic precursor which gels randomly. As discussed above, this successfully predicts high gel points but does not model the competition between different polycyclic and non-cyclic oligomers.

Fortunately, a technique has been developed which will allow us to model the formation of polycyclic species, by defining only a small number of reactions: dynamic Monte Carlo (DMC) simulations. This approach to simulating chemical dynamics was introduced by Gillespie $^{37}$ and brought to network polymerization by Dušek and coworkers. ${ }^{26,27}$ This method is well suited to systems such as nonideal polycondensation where correlations beyond those explicitly modeled in the kinetic expressions exist. ${ }^{28,29}$

This type of simulation can be interpreted as a way of solving the master equation for chemical dynamics, as a method of solving the differential equations for a set of coupled chemical reactions, or as a coarse-grained molecular simulation. The advantages of the technique have been discussed in more detail elsewhere. ${ }^{26-30,38}$ The equations literally being solved are analogous to Euler integration of a set of ordinary differential equations. However, instead of discretizing time into even intervals, we discretize reaction events (in this case, siloxane bond additions) and advance the simulation by even intervals of these events. By adding one siloxane bond per Monte Carlo step, we will advance the siloxane bond conversion, $\alpha=[$ SiOSi $] /[\text { SiOEt }]_{0}$, by a small constant increment (here, $\Delta \alpha=2.5 \cdot 10^{-6}$ for each Monte Carlo step).

If that were the only distinguishing feature of these simulations, they would not hold much interest over more advanced approaches to solving ordinary differential equation systems. What is unique about the Monte Carlo simulations is that, because each reaction happens 
between a specific pair of reacting sites, it is possible to keep track of extra information about the molecular weight distribution to which those sites belong, including membership in cyclic and polycyclic species. Therefore, we can bridge size scales from the local connectivity model embodied in the differential equations to the complete molecular size and shape distribution. Only the finite size of the population limits the maximum simulated length scale. As we will discuss, this also allows us to define local rates of forming cycles, and therefore to model the formation of polycyclic species in competition with molecular growth. By construction, DMC simulations (within finite size limitations) generate all oligomers required (and only those needed) by the differential equations being solved. This allows them to easily model pathways for formation of polycyclic species as in Figure 1.

Dynamic Monte Carlo simulations also hold more subtle advantages over other techniques that might be applied here. Because they are meso-scale models (not atomically detailed), they allow us to simulate much longer times and much larger ensembles than molecular dynamics simulations. ${ }^{7}$ Because each step of the Monte Carlo simulation is known to be a reaction event, no computational effort is wasted in diffusion toward unsuccessful reaction attempts. Also, while some aspects (such as neglecting diffusion effects, site accessibility limitations, and 3-dimensional packing constraints ${ }^{39}$ of the present simulations match statistical models and their perturbations, ${ }^{33,40}$ the DMC method accounts for the effects of the order (history) of bond addition to a polymer. This captures structural correlations beyond those explicitly written in the rate expressions. ${ }^{28-30}$ The work of Miller and coworkers has shown that those structural correlations are vital to a quantitatively accurate model..$^{28,29}$

Stepto and coworkers ${ }^{41}$ report using a related approach to model cyclization during network formation. However, they do not use the concentrations of bond blocks (see below) to define cyclization rates. Instead, they calculate the probability of intramolecular reaction at each condensation reaction by evaluating the distances from a randomly chosen site to all other sites in the molecule to which it belongs. This is equivalent to our approach only if there are no first-shell substitution effects. 


\section{The Model}

\section{Hydrolysis Pseudoequilibrium}

In the first steps of the network of reactions that occur during siloxane fabrication from alkoxysilanes, alkoxyl groups are hydrolyzed to generate hydroxyl groups. Experiments ${ }^{42,43}$ have shown that (under conditions yielding homogeneous gels at least) hydrolysis can reach pseudoequilibrium ${ }^{44}$ as described by the reaction in eq 1 :

$$
\equiv \mathrm{Si}-\mathrm{OR}+\mathrm{H}_{2} \mathrm{O} \stackrel{K_{k}}{\rightleftharpoons} \equiv \mathrm{Si}-\mathrm{OH}+\mathrm{ROH}
$$

where $R$ is an organic group, and $K_{h} \sim 18 \pm 10$ and is approximately independent of site identity. ${ }^{42}$ The overall fractional hydrolysis extent of hydrolysis $(\chi)$ is defined:

$$
\chi \equiv \frac{[\equiv \mathrm{SiOH}]}{[\equiv \mathrm{SiOH}]+[\equiv \mathrm{SiOR}]}
$$

The dependence of all condensation rates on the fractional hydrolysis extent is the same (see below), so hydrolysis affects only the absolute rate of reaction (not the results with respect to conversion). If water is the limiting reagent, the final extent of the reaction may also be determined by hydrolysis extent. We will not discuss hydrolysis further here except to mention that the dependence of $\chi$ on the instantaneous levels of water and alcohol is easily determined, ${ }^{44}$ and that the effects of competition between hydrolysis and condensation rates have been explored elsewhere. ${ }^{30}$

\section{Bimolecular Condensation}

Once sites have been hydrolyzed, they can react together to generate a new siloxane bond and water:

$$
\equiv S i-O H+H O-S i \equiv \longrightarrow \equiv S i-O-S i \equiv+H_{2} \mathrm{O}
$$

If the two silanols are attached to different oligomers, those oligomers react together by bimolecular condensation. We define the rate of bimolecular reaction between two sites 
based only on the connectivities of the sites (in other words, we restrict our attention to first-shell substitution effects for bimolecular reactions). Kinetic models using this type of substitution effect have been quite successful in matching ${ }^{29} \mathrm{Si}$ NMR data. ${ }^{23,32}$ If $Q_{i}$ represents a tetrafunctional silicon site with $i$ siloxane bonds, we can write bimolecular condensation as:

$$
Q_{i}+Q_{i^{\prime}} \stackrel{k_{i i^{\prime}}}{\longrightarrow} Q_{i+1}+Q_{i^{\prime}+1}+H_{2} O
$$

Where both $i$ and $i^{\prime}$ can vary between 0 and 3. This gives rise to a matrix of bimolecular reactions, which is represented in Figure 2.

We define condensation rate coefficients $k_{i i^{\prime}}$ on a per-silanol basis. The rate should be proportional to the product of the number of silanols present on each of the reacting sites, so:

$$
R_{i i^{\prime}}^{\text {bimol }}= \begin{cases}(f-i)\left(f-i^{\prime}\right) \chi^{2} k_{i i^{\prime}}\left[Q_{i}\right]\left[Q_{i^{\prime}}\right] & i \neq i^{\prime} \\ \frac{1}{2}(f-i)\left(f-i^{\prime}\right) \chi^{2} k_{i i^{\prime}}\left[Q_{i}\right]\left[Q_{i^{\prime}}\right] & i=i^{\prime}\end{cases}
$$

Condensation can also occur between a silanol and an ethoxy group with production of alcohol, but this route is negligible for ethoxysilanes. ${ }^{45,46}$ If alcohol-producing condensation were important, only the dependence of condensation rates on $\chi$ would change. ${ }^{23}$

The set of rate coefficients used in the simulation resembles the experimental substitution effect which for many ethoxysilanes under acidic conditions can be described by a large drop (we will use $90 \%$ ) in reactivity when the connectivity of both sites increases but a small drop (we will use $10 \%$ ) when the connectivity of only one site increases. ${ }^{23}$ Expressing $k_{i i^{\prime}}$ in matrix form:

$$
\mathrm{k}=k_{00} \cdot\left[\begin{array}{cccc}
1.0 & 0.9 & 0.81 & 0.729 \\
& 0.1 & 0.09 & 0.081 \\
& & 0.01 & 0.009 \\
& & & 0.001
\end{array}\right]
$$


We will always use this matrix for bimolecular reactions here (unless ideal polycondensation is simulated instead - in which case all condensation rate coefficients are equal and no cyclization will be allowed). The bimolecular condensation substitution effect may change with the $\mathrm{pH}$ of the reaction solution, ${ }^{31}$ with accompanying structural changes. ${ }^{47}$ We do not address these effects here.

\section{Cyclization}

One special capability of the Monte Carlo simulations is that without losing polymerization history information, we can define cyclization not only of isolated molecules but also of substructures within large molecules. We will use the concept of bond blocks for this. A bond block is a path, consisting of a number of bonds, between two sites in an oligomer (note that we will use the term "bond" to refer to a siloxane bond between silicon sites, which actually is a pair of chemical bonds ( $\mathrm{Si}-\mathrm{O}$ and $\mathrm{O}-\mathrm{Si}$ )). At the left in Figure 3, we show a two-bond block from silicon site $Q_{i}$ through a siloxane bond to another silicon site (a silicon site of any connectivity $\geq 2$ ) through a second siloxane bond to silicon site $Q_{i^{\prime}}$. This two-bond block will be represented as ${ }^{2} B_{i i^{\prime}}$ (the superscript referring to the number of bonds in the block and the subscripts to the connectivities of the terminal sites). Polymerization can be described entirely through the formation of larger and larger bond blocks but we will only use the concept here for cyclization. Since we are interested in the closure of all kinds of bond blocks, not just of bond blocks in linear oligomers (as modeled previously ${ }^{24}$ ), we allow unimolecular-like reactions as in Figure 3.

The rates for this set of reactions are given by:

$$
{ }^{2} R_{i i^{\prime}}^{c y c}=(f-i)\left(f-i^{\prime}\right) \chi^{2} k_{3 c\left(i, i^{\prime}\right)}\left[{ }^{2} B_{i i^{\prime}}\right]
$$

The only things limiting this approach are the ease of determining the concentrations of two-bond blocks (this is not trivial) and finding an appropriate bond when that reaction is chosen. Analogous expressions could be written for the rates of closure of bond blocks of any 
size $\left({ }^{n} R_{i i^{\prime}}^{c y c}\right)$, but we would not be able to simulate as large a system because the memory and computational requirements increase rapidly with bond block size. Instead, we focus here on the smallest known ${ }^{4,48}$ ring-forming (2-bond) blocks to start to learn about extensive cyclization. We will comment later on which features of silicate structure these simulations can actually capture.

The substitution effect for this set of cyclization reactions has never been measured experimentally. For the moment, we assume that the substitution effect for reaction between bond block-ends with different degrees of connectivity is the same as for bimolecular reactions between those sites:

$$
\mathrm{k}_{3 c}=k_{3 c(1,1)} \cdot\left[\begin{array}{ccc}
1.0 & 0.9 & 0.81 \\
& 0.1 & 0.09 \\
& & 0.01
\end{array}\right]
$$

In addition to resembling the experimental bimolecular condensation substitution effect, this matrix is also intuitively appealing because the closure of bond blocks in densely branched regions occurs more slowly than in less-condensed regions. By using this substitution effect, we also limit the number of adjustable parameters for the model. This is attractive at this early stage of exploring the capabilities of the simulation. One could always perform simulations with arbitrarily varying coefficients to assess the sensitivity of structure to the form of the rate coefficients. The purpose of this study is only to assess the model's predictions when our best guess of the experimental parameters is used.

To help us in examining the behavior of the system, we further define a dimensionless cyclization tendency $\kappa$ :

$$
\kappa \equiv \frac{k_{3 c(1,1)}}{k_{11}[S i]}
$$

This parameter is particularly useful because it contains the silicon concentration - an adjustable parameter. We previously found (and it is apparent from comparing the bimolecular and cyclization rate terms) that decreasing silicon concentration is equivalent to increasing 
all cyclization rate coefficients. ${ }^{24}$ This parameter has a lower bound defined by the maximum silicon concentration (given by the inverse molar volume of the siloxane mixture) but can be increased indefinitely by dilution. The bimolecular coefficient for reaction between singly connected sites is chosen for this coefficient because if only linear trimers were present, $\kappa$ would represent exactly the ratio of initial rates of the unimolecular cyclization reaction to that of bimolecular chain extension. This coefficient can be measured either by synthesizing the linear trimer ${ }^{48}$ or by following the reaction starting from a monomer. ${ }^{23}$

The challenge now is to find the bond block concentrations $\left\{\left[{ }^{2} B_{i i^{\prime}}\right]\right\}$. These can be determined, in principle, by calculating the distance matrix $\mathbf{D}^{49}$ of each molecule. Each element of $\mathbf{D}$ for a graph is the topological distance (path with the smallest number of bonds) between a pair of sites (i.e., all distinguishable bond blocks). Unfortunately, calculating $\mathbf{D}$ for the reactant and product molecules after each step in the simulation would be very expensive $e^{49}$ - especially as the gel point is approached and molecules of greater than $10^{3}$ sites are present. Therefore, we take a more efficient approach by only counting the new two-bond blocks created when a bond is added. This is the only place that bond blocks are generated.

To do this, we simply count the (up to) three nearest neighbors of each of the sites involved in each new bond (illustrated in Figure 4). New 2-bond blocks are formed between each of these neighbors and the other site forming the new bond. Up to six two-bond blocks can be created by one new bond. After each reaction, the program also updates the lists of bond blocks to account for the changing connectivity of existing blocks. If we were to consider larger bond blocks, not only would we need to find more distant neighbors from the members of each new bond (a procedure which scales as $(f-1)^{n-1}$ where $n$ is the block size), but we also would need to find all other new bond blocks crossing each newly created bond. For instance, up to nine new three-bond blocks are present in just the small fragment in Figure 4. 


\section{Simulation Procedure}

With the above method of determining bond block concentrations, all reactions occurring in the system are well defined. The simulation proceeds (as other DMC simulations of polycondensation ${ }^{24,27,30}$ ) by adding one new bond at each step. The probability that a particular type of polycondensation reaction will be chosen at a given step is proportional to its rate. After choosing a reaction, the sites participating in the reaction are randomly selected from the subset of sites matching the required characteristics (connectivity or bond block membership). So that cyclization reactions are well defined within this scheme, bimolecular reactions are only allowed between sites on differing molecules. Throughout the simulation, we maintain similar information to that described previously, ${ }^{24}$ including the set of neighbors of each site, lists of sites with varying numbers of siloxane bonds, the molecular weight distribution, the averages of the molecular weight, and the size of the largest oligomer.

Because reacting sites are chosen randomly, each realization is unique. However, if we choose a large enough system or iterate enough times, the average value of the concentrations in the differential equations (sites of differing connectivity and ring species in this case) can be very accurately determined. The characteristics of the population, such as the weightaverage degree of polymerization, ${ }^{38}$ are most accurately determined with the largest possible system size, within the limits of the allotted CPU time. ${ }^{50}$ In our case, 200,000 sites were used in simulations lasting between one and five hours on a Cray CR-90 machine.

Some new information is also maintained for the simulations performed here. We monitor the cycle rank (CR) distribution and the average cycle rank of the ensemble. The cycle rank is defined ${ }^{40}$ as the number of independent cycles that must be closed in order to form a given oligomer. In other words, it is the number of extra bonds present beyond that needed to connect all sites once. Mathematically,

$$
C R(x)=\frac{1}{2} \sum_{i=1}^{4} i Q_{i}(x)-D P(x)+1
$$

where $D P(x)$ is the number of mers in the oligomer $x$ and $Q_{i}(x)$ is the number of sites of 
connectivity $i$ in oligomer $x$. This definition is useful because it avoids arbitrary overcounting of paths. One must bear in mind that, like polymerization degree, $\mathrm{CR}$ is only unique to within isomerization. The "number average" cycle rank reported is defined:

$$
C R_{n}=\frac{\sum_{x=1}^{n} C R(x)}{n}=\frac{1}{2} D P_{n}\left(\sum_{i=0}^{f} i \phi_{i}-2\right)+1
$$

where $\phi_{i}$ is the fraction of sites of connectivity $i$.

The only other major change from the previously described Monte Carlo algorithm ${ }^{24}$ is in the way that cyclization is treated when those reactions are chosen. We keep an explicit list of all two-bond blocks capable of forming a ring, distinguished by the connectivities of their ends. When a cyclization reaction occurs, we randomly select a bond block from this list and the block is removed from the list. This convenience comes with a high cost of maintaining the list of bond blocks. Because of this, the program scales poorly with ensemble size. We are exploring alternative approaches.

\section{Results and Discussion}

Since the use of bond blocks is a new feature of this simulation, we first verified that we are properly accounting for the concentration of 2-bond blocks. A good case is ideal polycondensation, for which analytical expressions for the concentrations of all bond blocks can be derived. In the Supplemental Material we derive this expression and show that the Monte Carlo simulations of ideal polycondensation match this prediction up to and through gelation.

Once confident that the simulation counts two-bond blocks correctly, we tested the program's performance for ideal polycondensation and for FSSE only $(\kappa=0)$ against previous work $^{24,30,50}$ and found excellent agreement. Since those results agree with prior work, we do not present them here. 


\section{The Sol-Gel Transition}

The primary purpose of this investigation is to see how close this model - with coefficients matching early-conversion kinetic experiments - brings us to the experimentally measured gel conversion. Figure 5 shows how the weight average degree of polymerization $\left(D P_{w}=\right.$ $\sum_{x=1}^{n} D P(x)^{2} / \sum_{x=1}^{n} D P(x)$ where $D P(x)$ is the degree of polymerization of oligomer $x$ ) varies with conversion for different $\kappa$ values and for ideal polycondensation. The point at which $D P_{w}$ diverges gives one estimate of gel point. Other estimates can be found from the point at which the apparent gel fraction (the degree of polymerization of the largest molecule in the simulation over the total number of mers) appears to rise above zero, or the point at which the reduced weight average degree of polymerization (the sol $D P_{w}$ ) reaches its maximum value ${ }^{38}\left(D P_{w}^{r}\right.$ is calculated just as $D P_{w}$ is, but with the largest molecule (the "gel") excluded). The latter two definitions give identical results in this case and we show the estimated gel conversions in Figure 6.

The most remarkable feature of the values found is that this model is able to predict gel conversions that match or exceed the experimental gel conversion of $82 \%$ for tetraethoxysilane. ${ }^{18}$ This is the first alkoxysilane polymerization model able to extrapolate late-conversion structural features such as this gel conversion from early-conversion kinetic studies.

The one other model in Figure 6 which is able to match the experimental gel conversion is that due to Suematsu ${ }^{51}$ (eq (9) in the original paper). That model assumes independence of the conversion leading to gelation and that leading to cyclization and therefore only approximately simulates the competition between cyclization and growth. We can fit the first three points from the DMC simulations using the equation of Suematsu for $A_{f}$ polycondensation (recast in terms of $\kappa$ ), but the theory predicts that gelation should not be possible at a slightly higher $\kappa$. Suematsu's equation can predict neither the inflection point nor the plateau in $\alpha_{g e l}$ at high $\kappa$ values indicated by our simulations. We will return to this point later. 
The sol-gel transition does not always appear to be equally broad in Figure 5. We clarify this point in Figure 7, where the $D P_{w}^{r}$ is plotted as a function of the distance of the conversion from the gel point. Notice first that as $\kappa$ increases, the width of the peak first increases and then decreases. Also notice that the shape of the plot changes. At low $\kappa$ the plot is fairly symmetric, but has smaller peaks following the main peak. At intermediate $\kappa$ the sol molecular weight builds up quite a lot before gelation but drops precipitously thereafter. At even higher $\kappa$, the peak becomes more symmetric again as it sharpens.

The type of behavior displayed in Figure 7 can be divided into three regions depending on the cyclization tendency:

Low cyclization The tendency for cyclization is low enough that few rings form before gelation. For our model, this region is observed for $\kappa \leq 1.6$.

Competitive cyclization The cyclization rate increases with molecular weight, and in this regime, cyclization competes with cascading growth as the sol-gel transition begins. This results in a significantly broadened transition region. $D P_{w}^{r}$ rises gradually before the transition but once the gel finally appears, the sol molecular weight drops precipitously.

Precursor formation Cyclization is so fast that many cyclic species form before the solgel transition begins, leading to a distribution of precursor structures which undergo a narrow sol-gel transition. This regime has mostly been ignored in other modeling approaches. With our first shell substitution effect, this regime occurs for $\kappa \geq 10$.

\section{Relation to Other Models}

We already have compared the results of the present DMC simulations to a statistical gelation model including cyclization (Figure 6). That model assumes that " ... the change of functionality [during polycondensation] is not of primary importance in respect to the shift 
... "51 of the gel point. Further assuming a simplified dependence of cyclization rate on ring size gives the equation ${ }^{51}$ we have fit to the low- $\kappa$ data in the Figure. While the model is able to predict a high gel conversion, it incorrectly predicts that the ability to form a gel should be very sensitive to dilution.

Figure 6 shows that the model of Suematsu ${ }^{51}$ (and indeed most statistical models incorporating gelation approximately) account only for the first two regimes of cyclization limited cyclization and the competition between cyclization and growth. What they miss (and what happens to be vital for siloxanes) is the precursor formation regime. This regime may exist in other multifunctional polycondensation processes where, with high dilution, much cyclization occurs but functional groups remain which are still able to form a gel. It is also possible that in some cases, the amount of dilution required to enter this regime makes it impossible to form a sample-spanning gel, so precipitates form instead.

A method related to the current simulations but numerically more elegant is presented by Šomvársky, Dušek and Smrčková. ${ }^{52}$ Their Smoluchowski coagulation model uses a kernel accounting for the variation of, for instance, cyclization probability and steric effects during the course of polycondensation. The solution of the integrodifferential equations appearing in Smoluchowski coagulation and other population balance models has been extensively studied (e.g., by Ramkrishna and coworkers ${ }^{53}$ ).

The unimolecular kernels proposed in the past are likely to be useful in systems with limited amounts of cyclization, but we examine with the DMC model whether they can properly account for extensive cyclization. In the modeling of Šmvårsky et al. ${ }^{52}$ the rate of unimolecular reaction for a polymer $P_{n}$ of size $n$ is given by $K(n, l)\left[P_{n}\right]$ where $l$ is the number of functional groups available on this oligomer. They suggest that this kernel should be proportional to $l(l-1)$ (which is proportional to the number of pairwise combinations of functional groups on that oligomer). From our simulations, we can calculate $\langle l\rangle$, the average 
number of functional groups available per molecule:

$$
\langle l\rangle=\frac{\text { Number of functional groups }}{\text { Number of molecules }}=\frac{f[S i](1-\alpha)}{[S i] / D P_{n}}=f D P_{n}(1-\alpha)
$$

In our simulation, the unimolecular reaction rate depends on the numbers of 2-bond blocks present. Therefore, as an overall unimolecular reaction kernel, we use $K(n)=\left({ }^{2} B\right)_{n}$ $=$ the number of two-bond blocks in a polymer of size $n$. This form of kernel is implied in the Monte Carlo simulations of Dutton et al. ${ }^{41}$ In the Supplemental Material we derive an expression for the average number of two-bond blocks, $\left\langle{ }^{2} B\right\rangle$ as a function of conversion for ideal polycondensation. According to this equation, this value starts at zero and increases until it diverges at $\alpha=0.5$ (just like $D P_{n}$ ). In Figure 8 we plot the results of the ideal equation as a function of $\langle l\rangle(\langle l\rangle-1)$ as well as the values from our simulations.

For small $\kappa$ values, the curves look similar, but they are not linear. There is a slight downward deviation from the ideal curve due to the first-shell substitution effect. The curves bend upward as $\kappa$ increases because the functional groups per molecule are consumed at a faster rate than when $\kappa$ is small. As $\kappa$ increases further, the functional form changes a maximum in $\left\langle{ }^{2} B\right\rangle$ as a function of $\alpha$ occurs, so in Figure 8, a loop is observed for $\kappa=50$. While the curves in Figure 8 lie close to each other for at least some of the time, they are not all identical. Modifying the x-axis in Figure 8 as suggested by S̆omvársky et al. ${ }^{52}$ does not improve bring the curves any closer together. Therefore, it appears that the approach of Šomvársky et al. can be used to model this type of situation only approximately. More work will be needed to develop a unimolecular kernel which matches form of the curves in Figure 8 and accounts for loss of cycle-forming functional groups when cyclization occurs at a rapid rate.

\section{Relation to the Structure of Silicates}

We have shown that this model is capable of predicting gel conversions exceeding the experimental gel point of tetraethoxysilane. This is the first time that enough cyclization has 
been allowed to permit such a kinetic model to match this observable. This agreement is only a qualitative indication that we are moving in the right direction, however. The value of $\kappa$ at which the simulation line crosses the experimental gel conversion is 7. For 2 molar tetraethoxysilane, we know that for 3 -site rings only, $\kappa=1.05 .{ }^{48} \mathrm{On}$ the other hand, $\kappa$ is between 5 and 10 for 4 -site rings, ${ }^{17,23}$ so $\kappa=7$ may be a reasonable value for this over-simplified model.

Experimentally, gel points have only been measured for $\kappa$ varying by a factor of $1.86 .{ }^{18} \mathrm{In}$ the vicinity of the average experimental gel point in Figure 6, this gives a range of gel points (0.78-0.84) slightly larger than that observed experimentally (0.81-0.84). The experiments may be closer to the upper plateau of this curve, which would indicate that the simulated gel conversion at the upper plateau is too high. Including 4-site polycyclic species should lower the predicted gel conversion, though. ${ }^{18}$ One should also be concerned about how closely the simulated structures (with only three-site rings) resemble those of real silicates. Here, we discuss average structural indicators and compare them to the types of structures that have been observed in silicates.

Figure 9 shows the number average cycle rank as a function of the weight-average degree of polymerization. The cycle rank is minuscule when $\kappa$ is small. However, at high $\kappa$ values, $C R_{n}$ becomes quite large quickly. For instance, at $D P_{w}=10$ for the highest $\kappa$ value in the figure, $C R_{n}=5$. This number may not seem particularly high, but because the weightaverage degree of polymerization is biased towards high molecular weights, there actually may be smaller oligomers containing as many as five independent cycles.

Figure 10 shows an example of two of the structures present in the DMC ensemble which are of cycle rank five. One is the smallest possible oligomer - the pentacyclic pentamer - and the other is one example of a pentacyclic decamer. In both cases, the energetic penalties for creating molecules with so many three-site rings is large. Nothing like these structures has ever been observed in silicates or organically modified silicates. The closest oligomer to these that has been identified is a tetrahedral, tricyclic tetramer which has been 
claimed to be formed from sec-butyl trifunctional monomers. ${ }^{54}$ However, in ethoxy/hydroxysilicate solutions, the structures predicted by the simulation are probably too strained to be likely to exist. The assignment of a peak in ${ }^{29} \mathrm{Si}$ NMR of aqueous silicate solutions to the tetrahedral siloxane tetramer ${ }^{55}$ was subsequently ruled out by quantum mechanical calculations of energetic penalties ${ }^{10}$ and electronic effects. ${ }^{56}$

If the rings in these species were four-site rings, the degree of cyclization in these structures would be reasonable. For instance, the cycle rank of the octamer made up of four-site rings with one silicon site at each corner of a cube is five. This structure has not only been observed but can form in high yields under certain conditions. ${ }^{36}$ Other silicates with high levels of polycyclization have also been observed, so we conclude that the DMC model should be improved not by reducing the total amount of cyclization but instead allowing larger rings.

Another useful measure of the average level of cyclization in the population of oligomers is the ring involvement $(I)$, the average number of independent rings in which a silicon site is involved. It is defined as ratio of the expected number of ring memberships for a randomly selected site to the expected degree of polymerization of the oligomer to which a randomly selected site belongs. The expected number of ring memberships is just three times the expected cycle rank. For a single oligomer, the ring involvement is $3(\mathrm{CR} / \mathrm{DP})$. When calculating the average value, the weight averages are used, because they reflect the expected (average) values when a site is chosen random (rather than number averages, which are expectations for a randomly selected oligomer). Mathematically,

$$
I=\frac{\text { Total ring memberships }}{\text { Total sites }}=3 \frac{C R_{w}}{D P_{w}}=3 \frac{\sum_{x=1}^{n} C R(x) D P(x)}{\sum_{x=1}^{n} D P(x)^{2}}
$$

which is calculated periodically from the complete distribution of cycle ranks $\{C R(x)\}$ of the population in the simulation.

Figure 11 compares ring involvements from our DMC simulations with those calculated using our previous kinetic-recursive model with ring formation only from linear trimers. ${ }^{24}$ 
For this model, the concentrations of sites involved in one ring are known, so $I$ is just the ratio of the sum of the concentrations of all of these sites to the total site concentration. As $\kappa$ increases in the previous model, the ring involvement increases steadily up to a certain value, but plateaus as linear trimers disappear from the solution. The maximum observed ring involvement in this model is about 0.7 rings per silicon.

With the current model, on the other hand, Figure 11 shows that ring involvement increases steadily through gelation in the competitive cyclization region. For the precursorforming region $(\kappa=50)$, it appears that ring involvement does level off at about 2.7 rings per silicon site. This value approaches the level of three rings per site which we might expect in a silsesquioxane-like precursor. This level of cyclization, at least, is consistent with the ring involvement observed in silicates (oligomers with a ring involvement of up to three have been observed ${ }^{36}$ ). This result confirms the necessity of involvement of silicon sites in polycycles in order to have a quantitative model of polysiloxane structure evolution from alkoxysilanes. ${ }^{18}$

The next step in improving this model will be to allow four-site rings to form. Three-bond blocks will have to be determined to do this, which is conceptually similar to what has been done here but will require significantly more CPU time and memory. The kinetics of foursite ring formation significantly exceed those of three-site ring formation ${ }^{23}$ so this may be sufficient to bring the model into quantitative agreement with silicate gelation experiments. If not, it will be necessary to either introduce reversibility of rings to limit their number, or to limit a priori the involvement of each site in rings of different sizes. Both of these will be computationally intensive. The latter approach will require the definition of a number of rules not only about how bond blocks are counted, but also about how to remove bond blocks when a site is involved in a forbidden number of cycles. 


\section{Conclusions}

We have described a Monte Carlo simulation which incorporates known (or measurable) kinetics to predict siloxane structure development. The model is intended to approximate alkoxysilane polycondensation under acidic conditions. The three essential features modeled are hydrolysis pseudoequilibrium, a negative first-shell substitution effect for condensation reactions, and extensive cyclization with unimolecular-like rate terms. For computational expediency, the current model allows unlimited formation of 3-site rings only, which are not the most prevalent rings in silicates.

With a bimolecular reactivity trend taken from experiment and with cyclization coefficients assumed to follow a similar trend, the model is able to predict a gel conversion meeting or exceeding the unusually high gel conversion observed for tetraethoxysilane ( $82 \%)$. This is the first alkoxysilane polycondensation model sensitive to chemical effects which is able to predict such a high gel conversion. A dimensionless measure of the ratio of bimolecular condensation rate to ring closure rate $(\kappa)$ was defined. The simulations match the experimental gel conversion at $\kappa=7$. This is higher than the experimentally determined value of $\kappa$ for 3-membered ring formation in tetraethoxysilane, but may be consistent with the total level of cyclization in silica gelation.

Three regions were identified in our simulations, depending on the relative rate of cyclization compared to bimolecular condensation. At low cyclization rates, the sol-gel conversion resembles that of a model with first-shell substitution effects only. In principle, this regime can be reached by increasing the monomer concentration, but in practice, the necessary concentration may be higher than that of the neat monomer.

As the cyclization rate increases, we move into a region where cyclization and polymer growth are competitive for a large part of the process. Here, the sol-gel transition is broadened (the molecular weight increases more gradually) compared to the previous region because cyclization competes most effectively as the molecular weight of oligomers starts 
to diverge. This regime is implicit in other, statistical, models of gelation with cyclization. However, those models usually predict that gelation quickly becomes impossible because of consumption of all available functional groups through cyclization.

Instead, our simulations predict a limit to the number of functional groups that can be consumed by cyclization. Therefore as the cyclization rate increases yet further, a new regime emerges where cyclization and gelation are well separated. In this case, cyclization yields cage-like precursor oligomers ${ }^{18}$ which form prior to the onset of gelation. The molecular weight increases as rapidly as it did in the first regime (with no cyclization). This regime is absent from most statistical models of gelation with cyclization. The dependence of the number of 2-bond blocks is nonmonotonic with respect to the number of available pairs of functional groups on an oligomer. This may make it challenging to capture this regime with other theoretical or population balance approaches.

These simulations confirm our hypotheses that polycyclic or cage-like species 1) can be formed under kinetic trends consistent with experimentally observed rate coefficients and 2) are essential to explain the unusually high gelation conversion of tetraethoxysilane systems. Further improvement in the model are required before quantitative predictions can be made, however, by allowing larger rings to form.

\section{Acknowledgments}

The authors gratefully acknowledge the National Science Foundation and the University of Minnesota Graduate School for graduate fellowships to S.E.R., the National Science Foundation Center for Interfacial Engineering and Dow Corning Corporation for supporting this work, and the Minnesota Supercomputer Institute for a grant of supercomputer time. We also thank Dr. Gary Wieber of Dow Corning for helpful discussions.

\footnotetext{
Sandia is a multiprogram laboratory operated by Sandia Corporation, a Lockheed Martin Company, for the United States Department of Energy 


\section{References and Notes}

1. McGregor, R. R. Silicones and Their Uses; McGraw-Hill: New York, 1954.

2. Baney, R. H.; Itoh, M.; Sakakibara, A.; Suzuki, T. Chem. Rev. 1995, 95, 1409.

3. Bratton, G. J.; Currell, B. R.; Kendrick, D. A.; Midgley, H. G.; Parsonage, J. R. J. Mater. Chem. 1993, 3, 465.

4. Kelts, L. W.; Armstrong, N. J. J. Mater. Res. 1989, 4, 423.

5. Lux, P.; Brunet, F.; Virlet, J.; Cabane, B. Magn. Reson. Chem. 1996, 34, 173.

6. Brunet, F. J. Non-Cryst. Solids 1998, 231, 58.

7. Garofalini, S. H.; Martin, G. J. Phys. Chem. 1994, 98, 1311.

8. Clarson, S. J.; Semlyen, J. A. Siloxane Polymers; Prentice Hall: Englewood Cliffs, NJ, 1993.

9. West, J. K.; Zhu, B. F.; Cheng, Y. C.; Hench, L. L. J. Non-Cryst. Solids 1990, 121, 51.

10. Earley, C. W. J. Phys. Chem. 1994, 98, 8693.

11. Harrison, P. G. J. Organomet. Chem. 1997, 542, 141.

12. Wilcock, D. F. J. Am. Chem. Soc. 1947, 69, 477.

13. Tang, A.; Xu, R.; Li, S.; An, Y. J. Mater. Chem. 1993, 3, 893.

14. Loy, D. A.; Carpenter, J. P.; Myers, S. A.; Assink, R. A.; Small, J. H.; Greaves, J.; Shea, K. J. J. Am. Chem. Soc. 1996, 118, 8501.

15. Wada, M.; Kamiya, K.; Nasu, H. Phys. Chem. Glasses 1992, 33, 56. 
16. Kamiya, K.; Dohkai, T.; Wada, M.; Hashimoto, T.; Matsuoka, J.; Nasu, H. J. NonCryst. Solids 1998, 240, 202.

17. Ng, L. V.; McCormick, A. V. J. Phys. Chem. 1996, 100, 12517.

18. Ng, L. V.; Thompson, P.; Sanchez, J.; Macosko, C. W.; McCormick, A. V. Macromolecules 1995, $28,6471$.

19. Flory, P. J. J. Am. Chem. Soc. 1941, 63, 3083, 3091, 3096.

20. Stockmayer, W. H. J. Chem. Phys. 1943, 11, 45.

21. Stockmayer, W. H. J. Chem. Phys. 1943, 12, 125.

22. Klemperer, W. G.; Mainz, V. V.; Ramamurthi, S. D.; Rosenberg, F. S. Mat. Res. Soc. Symp. Proc. 1988, 121, 15.

23. Rankin, S. E.; Macosko, C. W.; McCormick, A. V. AIChE J. 1998, 44, 1141.

24. Kasehagen, L. J.; Rankin, S. E.; McCormick, A. V.; Macosko, C. W. Macromolecules 1997, 30, 3921 .

25. Rankin, S. E.; Macosko, C. W.; McCormick, A. V. Chem. Mater. 1998, 10, 2037.

26. Mikeš, J.; Dušek, K. Macromolecules 1982, 15, 93.

27. Šomvársky, J.; Dušek, K. Polym. Bull. 1994, 33, 369.

28. Sarmoria, C.; Vallés, E. M.; Miller, D. R. Macromolecules 1990, 23, 580.

29. Sarmoria, C.; Miller, D. R. Macromolecules 1991, 24, 1833.

30. Hendrickson, R. C.; Macosko, C. W.; Gupta, A. M. J. Comput. Poly. Sci. 1994, 4, 53.

31. Brinker, C. J.; Scherer, G. W. Sol-gel Science: The Physics and Chemistry of Sol-Gel Processing; Academic Press: Boston, 1990. 
32. Sanchez, J.; Rankin, S. E.; McCormick, A. V. Ind. Eng. Chem. Res. 1996, 34, 117.

33. Dušek, K. Recl. Trav. Chim. Pays-Bas 1991, 110, 507.

34. Bailey, J. K.; Macosko, C. W.; Mecartney, M. L. J. Non-Cryst. Solids 1990, 125, 208.

35. Stepto, R. F. T. Intra-molecular Reaction and Gelation in Condensation or Random Polymerisation. In Network Formation and Cyclisation in Polymer Reactions; Haward, R. N., Ed.; Developments in Polymerization 3; Applied Science Publishers: New Jersey, 1982.

36. McCormick, A. V.; Bell, A. T. Catal. Rev.-Sci. Eng. 1989, 31, 97.

37. Gillespie, D. T. J. Comp. Phys. 1976, 22, 403.

38. Gupta, A. M.; Hendrickson, R. C.; Macosko, C. W. J. Chem. Phys. 1991, 95, 2097.

39. On- and off- lattice simulations can capture these effects, ${ }^{38,57,58}$ but only at added computational complexity and expense which is not yet justified for this system.

40. Flory, P. J. Principles of Polymer Chemistry; Cornell University Press: Ithaca, NY, 1953.

41. Dutton, S.; Stepto, R. F. T.; Taylor, D. J. R. Angew. Makromol. Chem. 1996, 240, 39.

42. Rankin, S. E.; Šefčík, J.; McCormick, A. V. Ind. Eng. Chem. Res. 1999, 38, 3191.

43. Alam, T. M.; Assink, R. A.; Loy, D. A. Chem. Mater. 1996, 8, 2366.

44. Rankin, S. E.; McCormick, A. V. Chem. Eng. Sci. 2000, 55, 1955.

45. Assink, R. A.; Kay, B. D. Coll. Surf. A 1993, 74, 1.

46. Šefčík, J.; Rankin, S. E.; Kirchner, S. J.; McCormick, A. V. J. Non-Cryst. Solids 1999, $258,187$. 
47. Sanchez, J. Kinetics and Models of Silicon Alkoxide Polymerization, Thesis, University of Minnesota, 1994.

48. Sanchez, J.; McCormick, A. V. J. Non-Cryst. Solids 1994, 167, 289.

49. Rouvray, D. H. The Role of the Topological Distance Matrix in Chemistry. In Mathematics and Computational Concepts in Chemistry; Trinajstic, N., Ed.; Ellis Horwood Limited: Chichester, 1986.

50. Šomvársky, J.; Dušek, K. Polym. Bull. 1994, 33, 377.

51. Suematsu, K. Eur. Phys. J. B 1998, 6, 93.

52. Šomvársky, J.; Dušek, K.; Smrčková, M. Comp. Theor. Polym. Sci. 1998, 8, 201.

53. Ramkrishna, D. Rev. Chem. Eng. 1985, 3, 49.

54. Wiberg, V. E.; Simmler, W. Z. anorg. allg. Chemie 1955, 282, 330.

55. Kinrade, S. D.; Swaddle, T. W. Inorg. Chem. 1988, 27, 4253.

56. Moravetski, V.; Hill, J. R.; Eichler, U.; Cheetham, A. K.; Sauer, J. J. Am. Chem. Soc. 1996, 118, 13015 .

57. Anseth, K. S.; Bowman, C. N. Chem. Eng. Sci. 1994, 49, 2207.

58. Eichinger, B. E.; Akgiray, O. Computer simulation of polymer network formation. In Computer Simulation of Polymers; Colbourn, E. A., Ed.; Longman Science and Technology: Essex, 1994. 


\section{List of Figures}

1 Minimum reaction scheme needed to model the formation of the smallest known bicyclic silicate. Circles are silicon sites $\left[\mathrm{Si}\left(\mathrm{O}_{1 / 2}\right)_{4}\right]$ and lines are siloxane bonds between sites. Since all silicon sites are tetrafunctional, the remaining functional groups are capped by a combination of $\left(\mathrm{O}_{1 / 2} \mathrm{H}\right)$ (as illustrated for the monomer and dimer) and $\left(\mathrm{O}_{1 / 2} \mathrm{C}_{2} \mathrm{H}_{5}\right)$. Not all pathways for species disappearance are shown. . . . . . . . . . . . . . . .

2 Matrix of bimolecular reactions between sites of differing connectivity. Each cell contains a reaction between two silicon sites with varying number of siloxanes and uncondensed groups attached. To this figure there corresponds a symmetric matrix of condensation coefficients. . . . . . . . . . .

3 Cyclization reaction. The circle represents a site of any degree of condensation greater than or equal to two and lines represent siloxane bonds. Only the illustrated $(3-$ site $)$ rings are allowed to form. . . . . . . . . . . . .

4 Accounting procedure for two-bond segments. Circles are silicon sites and lines are siloxane bonds. The shaded circles are members of a new bond (thick line). At each condensation step, the new potential segments are counted from the site at one end of the new bond to each of the nearest neighbors of the site at

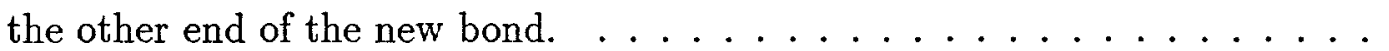

5 Simulated weight average degree of polymerization $\left(D P_{w}\right)$ as a function of conversion for varying $\kappa$ (cyclization tendency) from simulations of the polycondensation of 200,000 sites. For the ideal (dashed) curve, all rate coefficients are set equal to each other and $\kappa$ is set to zero. 
6 Gel conversion estimated from maximum value of $D P_{w}^{r}$ from polycondensation simulations with 200,000 sites as a function of $\kappa$. Dashed lines are benchmarks discussed in the modeling section. The dotted line is the best fit to the first three data of the equation suggested by Suematsu. ${ }^{51} \ldots \ldots \ldots \ldots$

$7 \quad$ Reduced weight average degree of polymerization $\left(D P_{w}^{r}\right)$ as a function of the distance from the gel conversion for simulations of 200,000 monomers with varying cyclization tendency $(\kappa) . \ldots \ldots \ldots \ldots \ldots$

8 A possible unimolecular cyclization kernel, the average number of 2-bond blocks, as a function of the average number of pairs of functional groups per molecule from our DMC simulations. . . . . . . . . . . . .

9 Simulated number-average cycle rank as a function of the weight-average degree of polymerization. All curves are Monte Carlo results. . . . . . . . . .

10 Examples of oligomers of cycle rank 5. Shown are two representative structures from the present simulations: (a) a decamer, and (b) a pentamer. . . 38

11 Average involvement of a silicon site in three-site rings as a function of conversion. Solid lines are results of our Monte Carlo simulations with unlimited cyclization. Dashed lines are from a kinetic-recursive model of polycondensation with isolated three-site ring formation from linear trimers only ${ }^{24} \ldots \ldots$ 


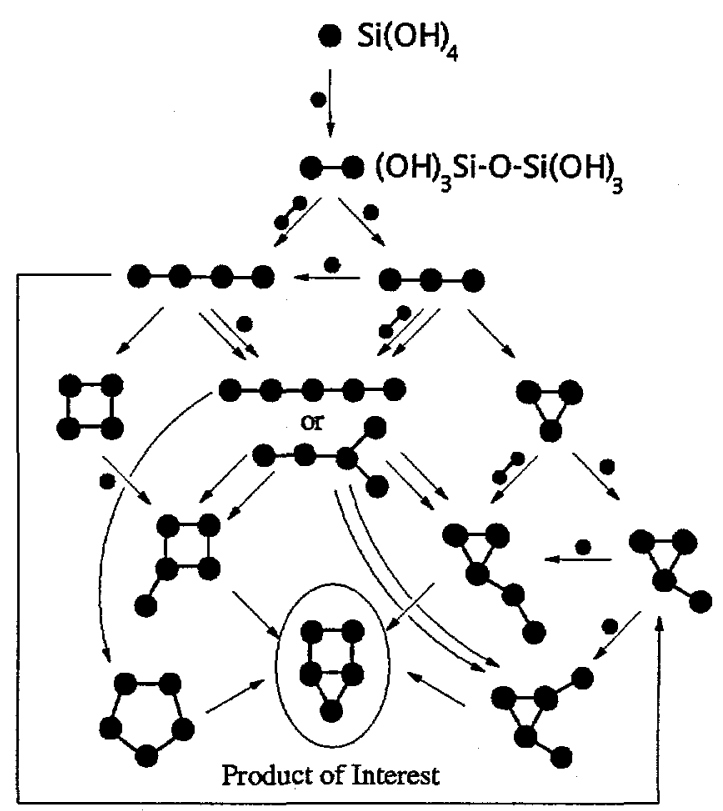

Figure 1: Minimum reaction scheme needed to model the formation of the smallest known bicyclic silicate. Circles are silicon sites $\left[\mathrm{Si}\left(\mathrm{O}_{1 / 2}\right)_{4}\right]$ and lines are siloxane bonds between sites. Since all silicon sites are tetrafunctional, the remaining functional groups are capped by a combination of $\left(\mathrm{O}_{1 / 2} \mathrm{H}\right)$ (as illustrated for the monomer and dimer) and $\left(\mathrm{O}_{1 / 2} \mathrm{C}_{2} \mathrm{H}_{5}\right)$. Not all pathways for species disappearance are shown. 


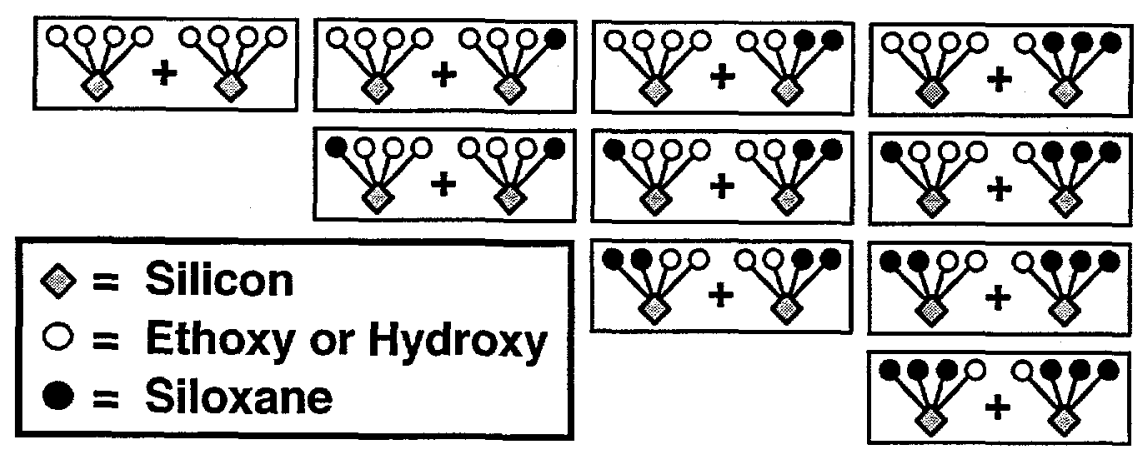

Figure 2: Matrix of bimolecular reactions between sites of differing connectivity. Each cell contains a reaction between two silicon sites with varying number of siloxanes and uncondensed groups attached. To this figure there corresponds a symmetric matrix of condensation coefficients. 


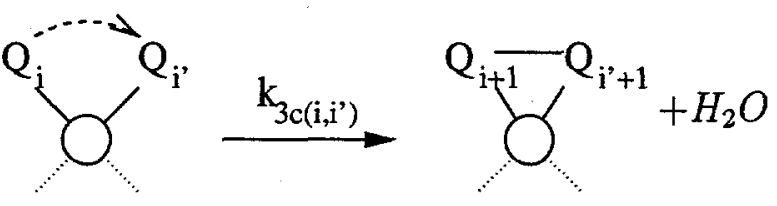

Figure 3: Cyclization reaction. The circle represents a site of any degree of condensation greater than or equal to two and lines represent siloxane bonds. Only the illustrated (3-site) rings are allowed to form. 


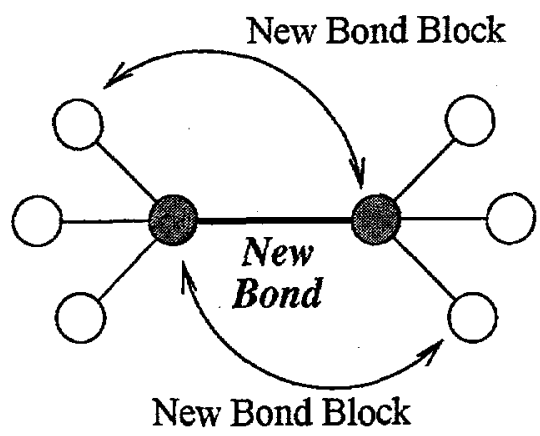

Figure 4: Accounting procedure for two-bond segments. Circles are silicon sites and lines are siloxane bonds. The shaded circles are members of a new bond (thick line). At each condensation step, the new potential segments are counted from the site at one end of the new bond to each of the nearest neighbors of the site at the other end of the new bond. 


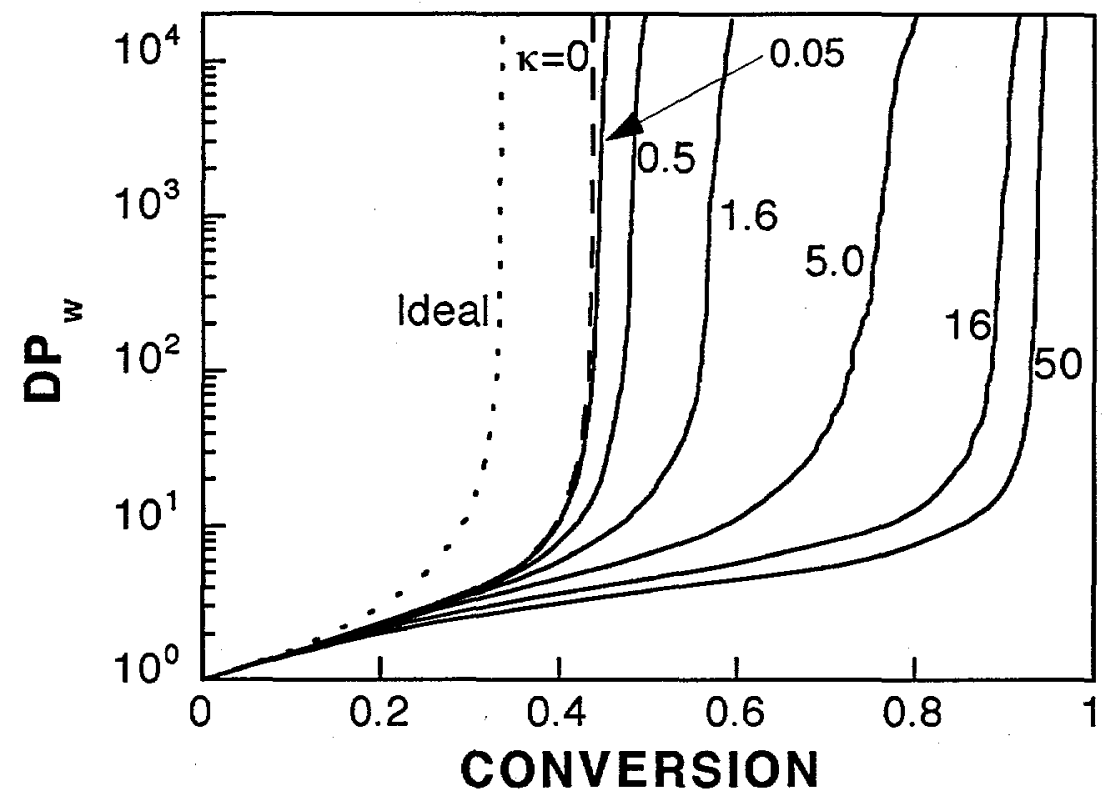

Figure 5: Simulated weight average degree of polymerization $\left(D P_{w}\right)$ as a function of conversion for varying $\kappa$ (cyclization tendency) from simulations of the polycondensation of 200,000 sites. For the ideal (dashed) curve, all rate coefficients are set equal to each other and $\kappa$ is set to zero. 


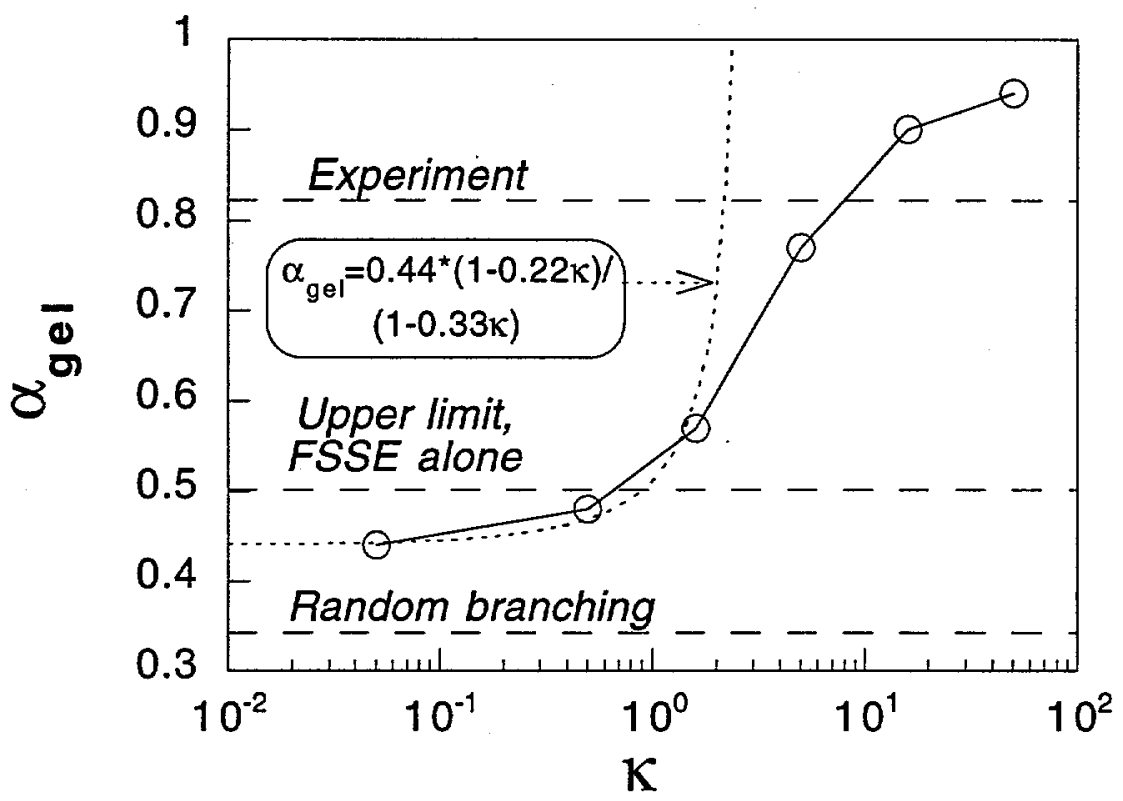

Figure 6: Gel conversion estimated from maximum value of $D P_{w}^{r}$ from polycondensation simulations with 200,000 sites as a function of $\kappa$. Dashed lines are benchmarks discussed in the modeling section. The dotted line is the best fit to the first three data of the equation suggested by Suematsu. ${ }^{51}$ 


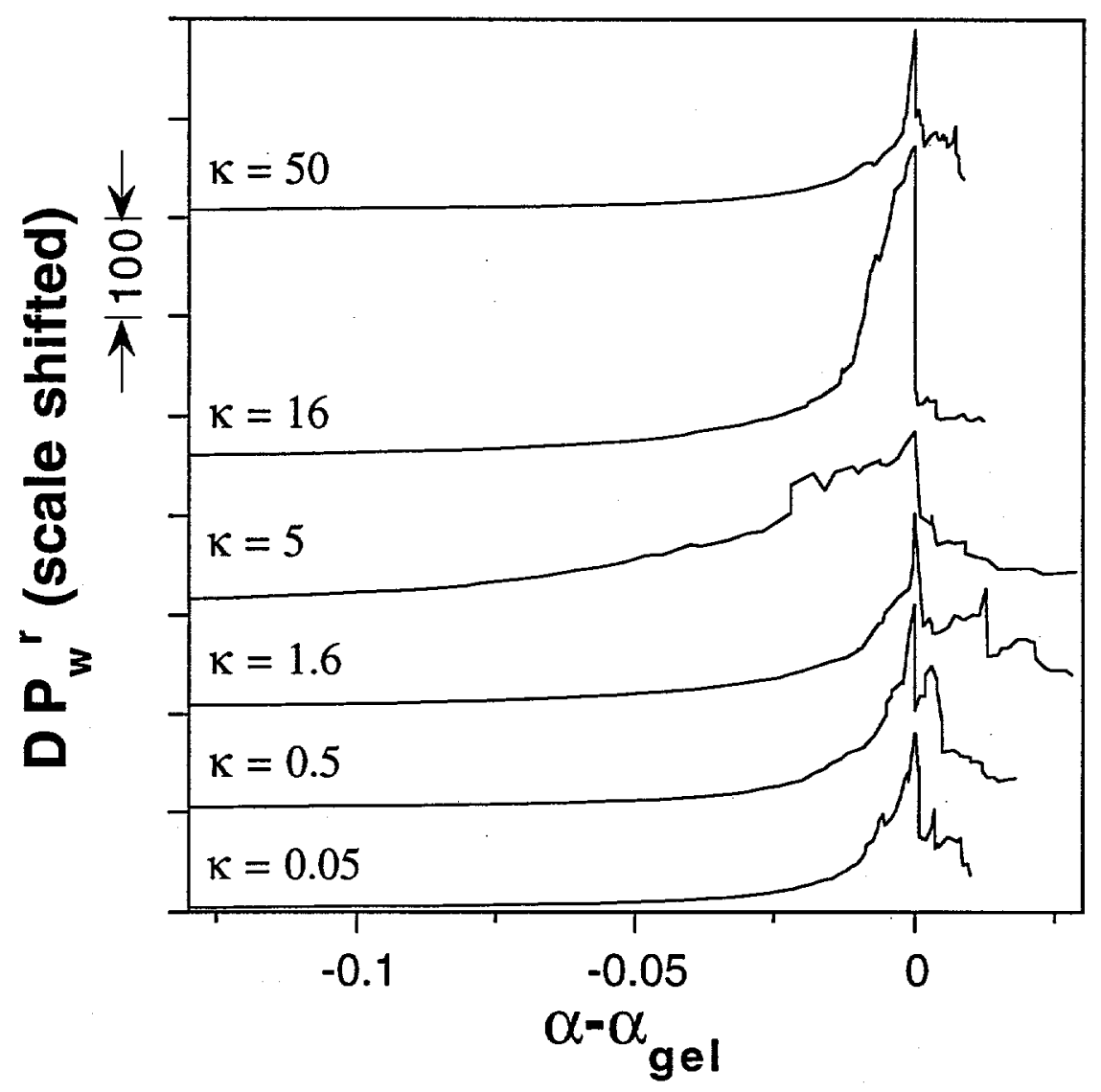

Figure 7: Reduced weight average degree of polymerization $\left(D P_{w}^{r}\right)$ as a function of the distance from the gel conversion for simulations of 200,000 monomers with varying cyclization tendency $(\kappa)$. 


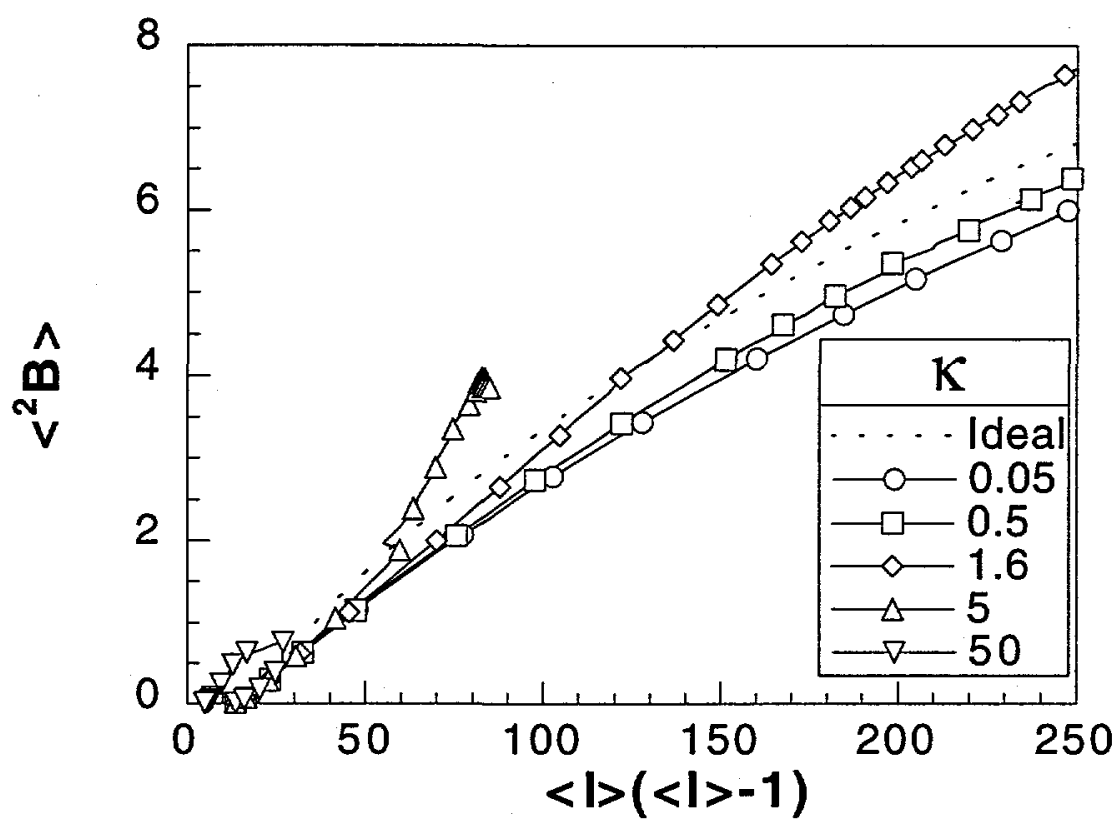

Figure 8: A possible unimolecular cyclization kernel, the average number of 2-bond blocks, as a function of the average number of pairs of functional groups per molecule from our DMC simulations. 


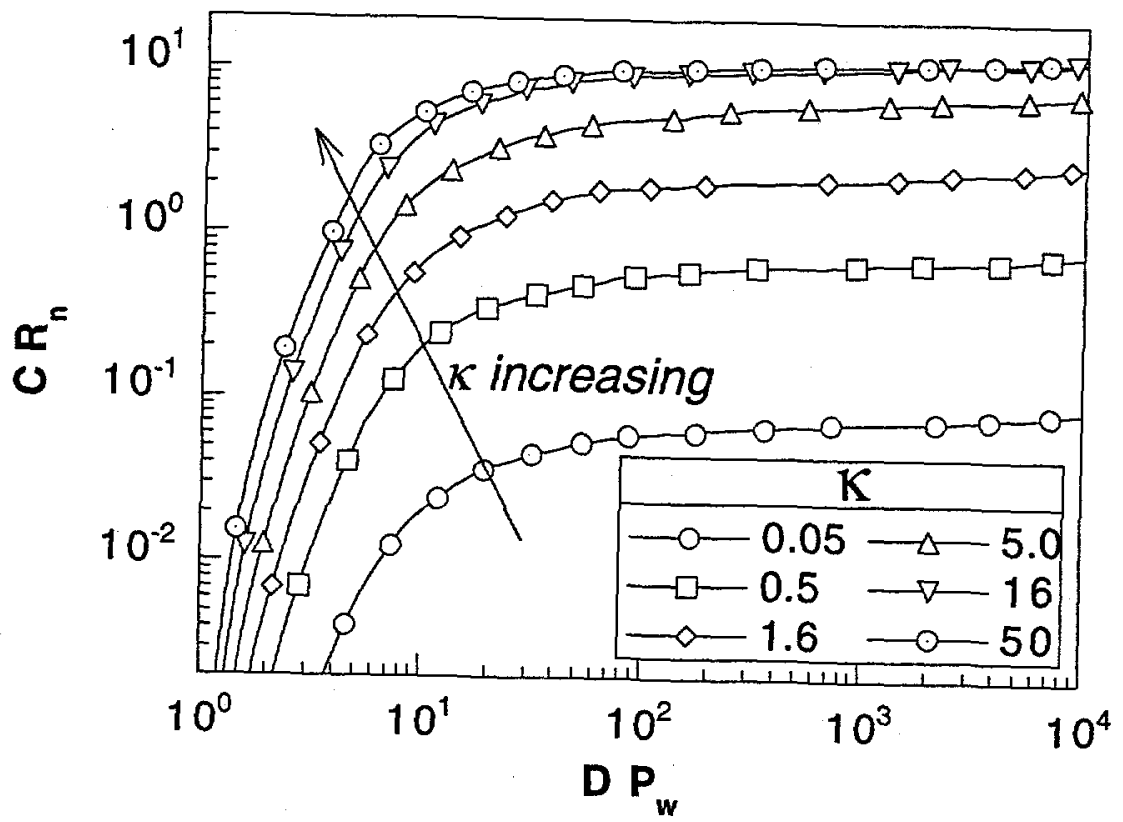

Figure 9: Simulated number-average cycle rank as a function of the weight-average degree of polymerization. All curves are Monte Carlo results. 
a)

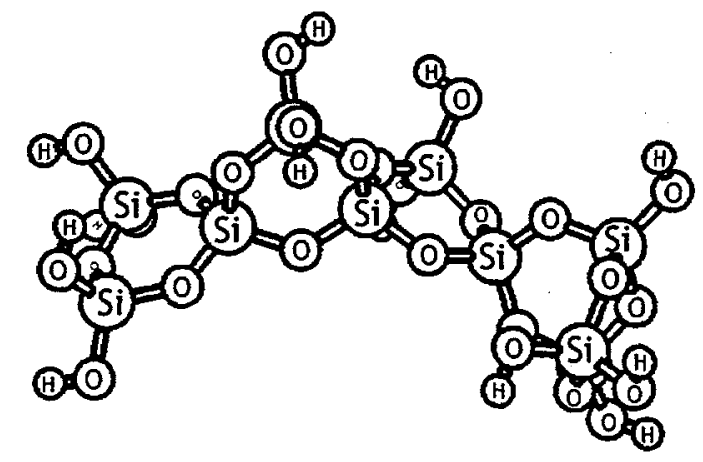

b) (1)

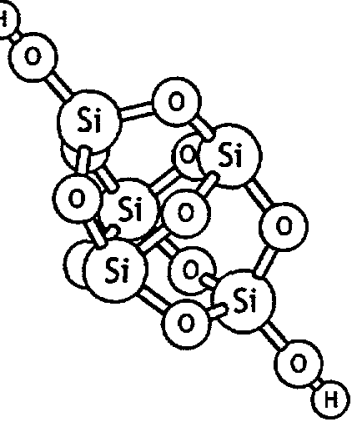

Figure 10: Examples of oligomers of cycle rank 5. Shown are two representative structures from the present simulations: (a) a decamer, and (b) a pentamer. 


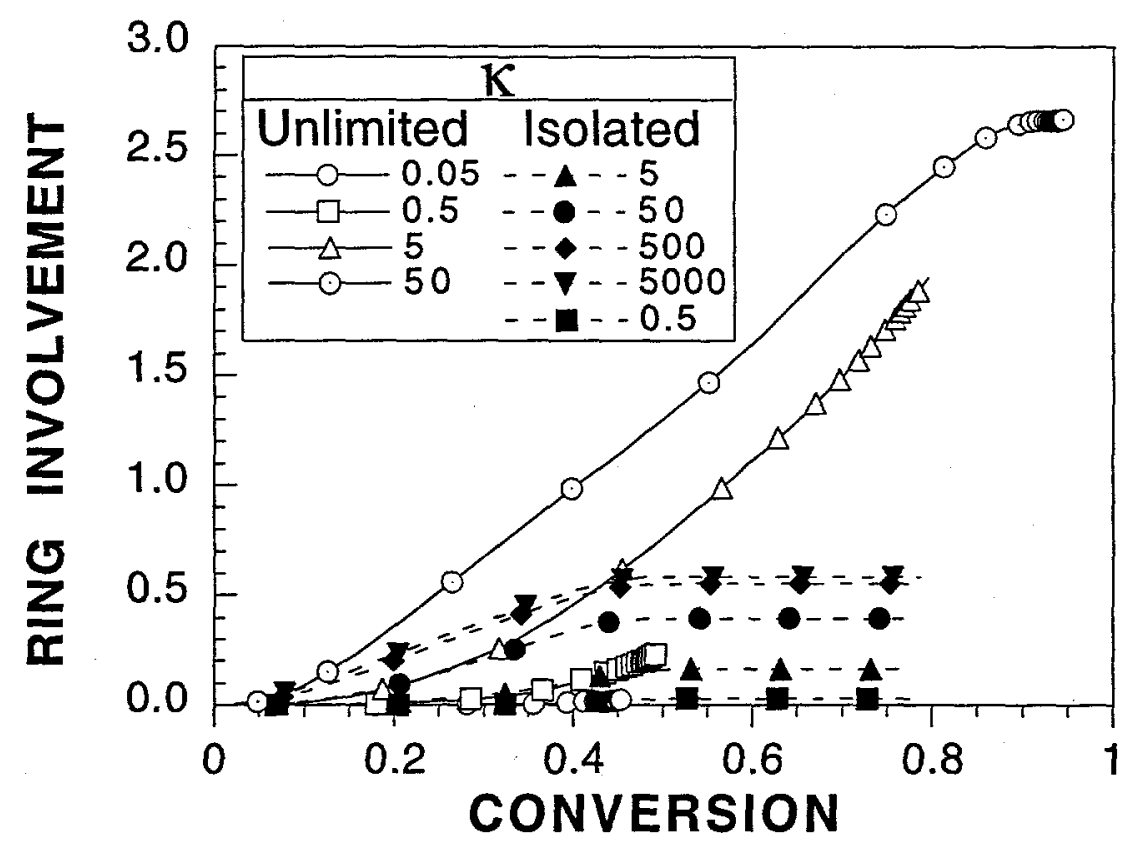

Figure 11: Average involvement of a silicon site in three-site rings as a function of conversion. Solid lines are results of our Monte Carlo simulations with unlimited cyclization. Dashed lines are from a kinetic-recursive model of polycondensation with isolated three-site ring formation from linear trimers only ${ }^{24}$. 